\title{
Méthodes particulaires régularisées. Développements récents et nouvelles applications
}

\author{
Jean Paul Vila*
}

\section{Résumé}

Dans cet article nous faisons une preśentation génerale des méthodes particulaires régularisées et plus particulièrement des méthodes SPH. Nous présentons également des travaux récents, basés sur une utilisation systématique des formulations faibles. Ils permettent d'introduire de nouveaux algorithmes, plus robustes et plus performants.

\section{Introduction}

Les méthodes particulaires régularisées pour les EDP ont fait l'objet de développements importants depuis les dix dernières années tant sur le plan des applications que de leur analyse numérique.

Beaucoup d'applications concernent la mécanique des fluides et l'on doit en particulier citer les méthodes de Vortex pour la dynamique des fluides incompressibles (visqueux ou non). Les méthodes de type PIC (Particle in Cell) sont également connues pour leurs applications à la dynamique des gaz compressibles. Elles partagent avec les méthodes de Vortex la caractéristique de faire intervenir une méthode numérique spécifique où mathématique pour traiter les termes différentiels qui ne sont pas des termes de transport:

- noyaux de Green pour les méthodes de Vortex

- couplage particule grille fixe pour les méthodes PIC

Dans cet exposé on s'intéresse à une autre classe de méthodes dont le principe est l'utilisation exclusive des approximations particulaires (régularisées) pour l'estimation des termes différentiels de l'EDP. Les aspects liés aux méthodes particulaires pour les équations de Boltzmann, Vlasov et similaires sont également exclus du cadre étudié ici.

La méthode SPH (Smooth Particle Hydrodynamics) est à la base des développements autour du thème abordé dans cet exposé. Nous commençons donc par un bref historique.

La méthode a été découverte en 1977 par Lucy ([17]) un astrophysicien anglais, son interprétation était cependant de nature probabiliste et les calculs utilisaient moins de 100 particules. C'est à J. Monaghan, mathématicien appliqué australien, que l'on doit les fondements effectifs de la méthode ([7], [8], [19]). Il est également à l'origine des extensions de la méthode au cadre multifluide, à la MHD ainsi qu' à un bon nombre des astuces qui en font une méthode opérationnelle. La méthode SPH est restée jusque dans les années 85 l'apanage des astrophysiciens. On doit à $W$. Benz (encore un astronome) les premières applications à des calculs complexes faisant intervenir des modèles d'endommagement de matériaux ([5],[6]). Ce type de modèle a éte ensuite utilisé par de nombreux centres de recherches pour les applications en dynamique rapide ([23], [11]). Les premiers codes industriels utilisant la méthode SPH ne sont apparus que récemment. Une des principales raisons est le manque de conceptualisation dans la formulation des conditions aux limites, qui ne permet d'offrir la souplesse attendue par un code Volumes Finis ou Eléments Finis.

*Mathématiques pour l'Industrie et la Physique. INSAT Département GMM. vila@mip.ups-tlse.fr 
Côté mathématique, et jusqu'a très récemment le seul travail d'analyse est du à Raviart Mas Gallic ([18],1987), il concerne la convergence de la méthode dans le cas continu en temps et pour les systèmes linéaires symétriques. Dans ce qui suit je vais décrire quelque travaux effectués au laboratoire MIP à Toulouse qui, me semblent il, ont fait progresser l'analyse de la méthode. Il faut également mentionner les travaux récents de Perthame.

Je donne d'abord un aperçu des principes classiques qui ont permis de développer les méthodes SPH. Je développe ensuite une nouvelle approche basée sur une formulation faible qui permet de mieux appréhender différents problèmes de nature théorique ou numériques liés à ces méthodes, en particulier sur les aspects longueur de lissage variable (tout a fait essentiels dans les applications) et leurs liens avec la conservativité et la consistance de la méthode.

\section{Principes Classiques}

Nous donnons brièvement dans ce qui suit les principes de bases nécessaires à la compréhension des méthodes particulaires. Nous renvoyons en particulier à l'ouvrage de P. A. Raviart ([25]) pour une description détaillée et des résultats mathématiques précis.

\subsection{Approximation Particulaire des Fonctions}

Les méthodes particulaires reposent sur des formules de quadraturesur des particules mobiles $\left(x_{i}(t), w_{i}(t)\right)_{i \in P} . P$ est l'esemble des particules, $x_{i}(t)$ est la position de la particule $i$ qui est déplacée le long des caractéristiques du champ $v$ (supposé régulier), alors que $w_{i}(t)$ est le poids (ou volume attaché à cette particule) et évolue selon div $v$ afin de conserver le volume:

$$
\begin{aligned}
\frac{d}{d t} x_{i} & =v\left(x_{i}, t\right) & \frac{d}{d t} w_{i} & =\operatorname{div}\left(v\left(x_{i}, t\right)\right) w_{i} \\
x_{i}(0) & =\xi_{i} & w_{i}(0) & =\omega_{i}
\end{aligned}
$$

$\left(\xi_{i}, t\right)$ sont les coordonnées Lagrangiennes de la particule $i$. On a ainsi les formules de quadrature suivantes où $J(\xi, t)$ est le jacobien de la transformation $(\xi, t) \rightarrow(x, t)$ :

$$
\int_{\mathbb{R}^{d}} f(x) d x=\int_{\mathbb{R}^{d}} f(x(\xi, t)) J(\xi, t) d \xi \approx \sum_{j \in P} w_{j}(t) f\left(x_{j}(t)\right)
$$

Approximation particulaire régularisée:

La formule de quadrature précédente, associée aux deux ingrédients suivants:

- noyau régularisant,

- régularisation par convolution,

permet de définir en trois étapes l'approximation particulaire régularisée d'une fonction:

- Noyau régularisant $W(x, h)$

$$
W(x, h)=\frac{1}{h^{d}} \theta\left(\frac{\|x\|}{h}\right)
$$

Il est définit par la donnée d'une fonction $\theta$ régulière à support compact $\subset[0,2]$ ( $d$ est la dimension de l'espace), par exemple:

$$
\theta(y)=C \times \begin{cases}1-\frac{3}{2} y^{2}+\frac{3}{4} y^{3} & \text { if } 0 \leq y \leq 1 \\ \frac{1}{4}(2-y)^{3} & \text { if } 1 \leq y \leq 2\end{cases}
$$


$C=\frac{2}{3}, \frac{10}{7 \pi}, \frac{1}{\pi}(\mathrm{d}=1,2$ ou 3$) . W(x, h) \rightarrow \delta$ quand $h \rightarrow 0 . h$ est la longueur de lissage ("smoothing length" dans le jargon de la communauté SPH).

- Régularisation de $f$

Elle se fait par convolution de $f$ par le noyau $W$ :

$$
<f(x)>=f * W(x)=\int f(y) W(x-y, h) d y
$$

- Quadrature de (2)

On effectue alors une quadrature de l'intégrale dans (2) avec la formule (1), ce qui définit l'approximation particulaire régularisée $\Pi^{h}(f)$ de la fonction $f$ :

$$
\Pi^{h}(f)(x)=\sum_{j \in P} w_{j} f\left(x_{j}\right) W\left(x-x_{j}(t), h\right)
$$

On utilise les notations suivantes:

$$
W_{i j} \equiv W\left(x_{j}-x_{i}, h\right) \quad \nabla W_{i j} \equiv \operatorname{grad}_{x}\left[W\left(x_{i}-x_{j}, h\right)\right]
$$

La dérivée de $f$ est approchée par la dérivée $\nabla \Pi^{h}(f)$ de $\Pi^{h}(f)$ définie par:

$$
\nabla \Pi^{h}(f)_{i}=\sum_{j \in P} w_{j} f\left(x_{j}\right) \nabla W_{i j}
$$

Des estimations d'erreur précises sont connues, elles ont été en particulier obtenues par P.A. Raviart, S. Mas Gallic et G.H. Cottet). Dans le cas d'un noyau $W \in C^{m+1}, m \geq 2$ et à support compact on a:

$$
\begin{aligned}
& \forall u \in W^{\mu, p}\left(\mathbb{R}^{d}\right), \quad s \geq 0, \mu=\max (r+s, m), \\
& \quad r=1,2, \frac{d}{m} \leq p \leq \infty, \quad q=\frac{p-1}{p} \\
& \left|u-\Pi^{h}(u)\right|_{s, p, \mathbb{R}^{d}} \leq \\
& \quad C\left(h^{r}|u|_{r+s, p, \mathbb{R}^{d}}+\left(1+\frac{\Delta x}{h}\right)^{\frac{d}{q}} \frac{(\Delta x)^{m}}{h^{m+s}}\|u\|_{m, p, \mathbb{R}^{d}}\right)
\end{aligned}
$$

Noter bien qu'il est nécessaire que le rapport $\frac{(\Delta x)}{h} \longrightarrow 0$, pour que $\Pi^{h}(u) \rightarrow u$. Dans ces formules $\Delta x$ est l'échelle caractéristique de la distance moyenne entre particule. Ceci signifie - W étant à support compact - que le nombre de particules voisines (au sens des particules intervenant effectivement dans les sommes définissant $\Pi^{h}(u)$ et $\nabla \Pi^{h}(u)$ ) doit tendre vers l'infini quand les pas de discrétisation $h$ et $\Delta x$ tendent vers 0 .

\subsection{Approximation Particulaire d'une loi de Conservation}

Je présente ici les concepts sur lesquels sont basés la méthode SPH initialement proposée dans $([17],[8])$. On introduit ces principes sur une loi de conservation modèle:

$$
L_{v}(\Phi)+\operatorname{div} F(x, t, \Phi)=S
$$

où $L_{v}(\Phi)$ est l'opérateur de transport:

$$
L_{v}(\Phi)=\frac{\partial \Phi}{\partial t}+\sum_{l=1, d} \frac{\partial}{\partial x^{l}}\left(v^{l} \Phi\right)
$$


Pour l'analyse mathématique de la convergence, $v$ est supposé connu et indépendant de $\Phi$, mais rien n'empêche formellement une telle dépendance dans tout ce qui suit. On recherche donc une approximation $\left(\Phi_{i}(t)\right)_{i \in P}$ de (4) définie sur des particules se déplaçant selon les caractéristiques de $v$ :

$$
\Phi_{i}:=\Phi\left(x_{i}(t), t\right)
$$

On a alors

$$
\frac{1}{w_{i}} \frac{d}{d t}\left(w_{i} \Phi_{i}\right)=L_{v}(\Phi)_{i}
$$

Pour calculer de manière approchée div $F(x, t, \Phi)$, il est naturel de régulariser $F$, ce qui donne alors pour l'approximation de (4):

$$
L_{\imath}(\Phi)_{i}+\nabla \Pi^{h}(F)_{i}+F_{i} \nabla \Pi^{h}(1)_{i}=S_{i}
$$

soit

$$
\frac{d}{d t}\left(w_{i} \Phi_{i}\right)+w_{i} \sum_{j \in P} w_{j}\left(F_{j}+F_{i}\right) \nabla W_{i j}=w_{i} S_{i}
$$

Le terme $\nabla \Pi^{h}(1)_{i}$ a été rajouté afin de préserver la conservation globale de la méthode. On remarque d'abord qu'il ne doit pas perturber la consistance de la méthode puisqu'il est a priori petit (d'après (3)), on remarque ensuite que $\nabla W_{i j}=-\nabla W_{j i}$, un calcul simple montre alors que:

$$
\frac{d}{d t}\left(\sum_{i \in P} w_{i} \Phi_{i}\right)=\sum_{i \in P} w_{i} S_{i}
$$

analogue discret de la relation traduisant la conservation globale de $\Phi$ :

$$
\frac{d}{d t}\left(\int_{\mathbb{R}^{d}} \Phi d x\right)=\int_{\mathbb{R}^{d}} S d x
$$

Il est à noter que (6) est un schéma de type centré, il y a donc nécessité de viscosité artificielle ou de décentrement pour stabiliser la méthode. Ceci est fait en pratique en introduisant un terme approprié $\Pi_{i j}$ (symétrique en $i$ et $j$ afin de respecter la conservativité globale ):

$$
\frac{d}{d t}\left(w_{i} \Phi_{i}\right)+w_{i} \sum_{j \in P} w_{j}\left(F_{j}+F_{i}+\Pi_{i j}\right) \nabla W_{i j}=w_{i} S_{i}
$$

Il reste a effectuer une discrétisation en temps explicite pour obtenir un schéma opérationnel.

\subsection{Applications aux équations d'Euler}

Nous appliquons maintenant ces concepts aux équations d'Euler de la dynamique des gaz compressibles pour lesquelles la méthode a été initialement développée. Les équations ont la forme suivante en dimension deux d'espace:

$$
\begin{gathered}
\Phi=\left(\begin{array}{c}
\rho \\
\rho v^{1} \\
\rho v^{2} \\
E
\end{array}\right) \quad F^{1}(\Phi)=\left(\begin{array}{c}
0 \\
p \\
0 \\
v^{1} p
\end{array}\right) \quad F^{2}(\Phi)=\left(\begin{array}{c}
0 \\
0 \\
p \\
v^{2} p
\end{array}\right) \\
p=p(\rho, u) \quad E=\rho\left(u+\frac{1}{2}\|\vec{v}\|^{2}\right) \\
L_{v}(\Phi)+\sum_{l=1, d}\left(\frac{\partial \Pi^{h}\left(F^{l}(\Phi)\right)}{\partial x^{l}}\right)=0
\end{gathered}
$$

ESAIM: Proc., VOL. 3, 1998, 131-146 
L’approximation particulaire SPH est alors définie selon:

$$
L_{v}(\Phi)_{i}+\sum_{l=1, d} F^{l}(\Phi)_{i}\left(\frac{\partial \Pi^{h}(1)}{\partial x^{l}}\right)+\left(\frac{\partial \Pi^{h}\left(F^{l}(\Phi)\right)}{\partial x^{l}}\right)_{i}=0
$$

ce qui conduit au système d'équations différentielles ordinaires:

$$
\begin{aligned}
& \text { (i) } \frac{d \overrightarrow{x_{i}}}{d t}=\overrightarrow{v_{i}}, \quad(\text { ii }) \quad \frac{d}{d t}\left(w_{i} \rho_{i}\right)=0 \\
& \text { (iii) } \frac{d}{d t}\left(w_{i} \rho_{i} \overrightarrow{v_{i}}\right)+w_{i} \sum_{j \in P} w_{j}\left(p_{i}+p_{j}\right) \nabla W_{i j}=0 \\
& \text { (iv) } \frac{d}{d t}\left(w_{i} E_{i}\right)+w_{i} \sum_{j \in P} w_{j}\left(p_{i} \overrightarrow{v_{i}}+p_{j} \overrightarrow{v_{j}}\right) \cdot \nabla W_{i j}=0
\end{aligned}
$$

$(i i) \Longrightarrow w_{i}(t) \rho_{i}(t)=c s t \equiv m_{i}$

$m_{i}$ est donc interprétée comme la masse d'une particule, conservée au cours du temps et donc caractéristique d'une méthode Lagrangienne.

$$
\left\{\begin{aligned}
\frac{d}{d t}\left(\overrightarrow{v_{i}}\right) & =-\sum_{j \in P} m_{j}\left(\frac{p_{i}+p_{j}}{\rho_{i} \rho_{j}}\right) \vec{\nabla} W_{i j} \\
\frac{d}{d t}\left(u_{i}\right) & =-\sum_{j \in P} m_{j} \frac{p_{j}}{\rho_{i} \rho_{j}}\left(\overrightarrow{v_{j}}-\overrightarrow{v_{i}}\right) \cdot \vec{\nabla} W_{i j}
\end{aligned}\right.
$$

La viscosité artificielle est introduite suivant le bon vieux principe de Von Neumann Richtmeyer $([27]): p \longrightarrow p+\Pi_{v}$ avec

$$
\Pi_{v}= \begin{cases}\beta \rho l^{2}(\operatorname{div} \vec{v})^{2}-\alpha \rho l c \operatorname{div} \vec{v} & \text { si } \operatorname{div} \vec{v}<0 \\ 0 & \text { sinon }\end{cases}
$$

où $\alpha$ et $\beta$ sont des coefficients adimensionnels positifs de l'ordre de l'unité.

Les équations (8) ne sont pas les équations que l'on trouve traditionnellement dans la littérature $\mathrm{SPH}$, on trouve le plus souvent la formulation suivante:

$$
\begin{gathered}
\left\{\begin{array}{l}
\frac{d}{d t}\left(\overrightarrow{v_{i}}\right)=-\sum_{j \in P} m_{j}\left(\frac{p_{i}}{\rho_{i}^{2}}+\frac{p_{j}}{\rho_{j}^{2}}+\Pi_{i j}\right) \nabla W_{i j} \\
\frac{d}{d t}\left(u_{i}\right)=-\frac{p_{i}}{\rho_{i}^{2}} \sum_{j \in P} m_{j}\left(\overrightarrow{v_{j}}-\overrightarrow{v_{i}}\right) \cdot \nabla W_{i j} \\
-\frac{1}{2} \sum_{j \in P} m_{j} \Pi_{i j}\left(\overrightarrow{v_{j}}-\overrightarrow{v_{i}}\right) \cdot \nabla W_{i j}
\end{array}\right. \\
\Pi_{i j}= \begin{cases}\frac{\mu_{i j}\left(\beta \mu_{i j}-\alpha \bar{c}\right)}{1 / 2\left(\rho_{i}+\rho_{j}\right)} & \operatorname{si}\left(\vec{v}_{i}-\vec{v}_{j}\right) \cdot\left(\vec{x}_{i}-\vec{x}_{j}\right)<0, \\
0 & \operatorname{sinon}\end{cases}
\end{gathered}
$$

où $\bar{c}=1 / 2\left(c_{i}+c_{j}\right)$ est la vitesse du son moyenne et $\mu_{i j}$ une approximation de $l$ div $\vec{v}$ :

$$
\mu_{i j}=\frac{h\left(\vec{v}_{i}-\vec{v}_{j}\right) \cdot\left(\vec{x}_{i}-\vec{x}_{j}\right)}{\left|\vec{x}_{i}-\vec{x}_{j}\right|^{2}+\epsilon h^{2}}
$$

Cette formulation peut se déduire de principes très voisins de ceux que je viens d'énoncer, je renvois à [26] (voir également [31] pour une présentation générale) pour de plus amples détails. 


\subsection{Commentaires}

Discrétisation en temps Les codes SPH utilisent le plus souvent des discrétisations en temps de type Runge et Kutta, et sont soumises de fait à des conditions de stabilité de type CFL.

Structures de Données. Coût Les codes SPH de part leur principe particulaire, n'ont pas besoin de structure de données lourde liée au maillage de particules. Il est cependant nécessaire de calculer à chaque pas de temps un minimum de données structurelles. Il s'agit de la liste des voisins d'une particule donnée $i$ intervenant dans le calcul des sommes de (7). Dans les codes usuels où n'interviennent pas de forces à longue portée (comme les forces gravitationnelles en astrophysique) la solution la plus efficace semble les algorithmes de "linked list", en terme de coût CPU (sur machine séquentielle) le coût de ces recherches est inférieur à $50 \%$ du coût d'un pas de temps. Des algorithmes basés sur des structures d'arbres (cf. [10]) sont utilisés dans les situations plus complexes, ils s'avèrent néanmoins trop coûteux dans les situations usuelles où le nombre de voisins effectifs ne dépasse pas 25 en dimension 2 et 70 en dimension trois. Pour donner des éléments de comparaison un code Volumes Finis d'ordre 2 sur maillage non structuré fonctionne avec au moins trois fois moins de voisin, au prix cependant d'une structure de données plus lourde (compensée par l'absence d'algorithmes de recherche de voisins). Par rapport à un code eulérien standard, un code SPH peut être actuellement de l'ordre de 2 à trois fois plus cher (i.e. moins rapide), il permet cependant d'effectuer des calculs Lagrangiens sans les déboires habituels liés en particulier aux remaillages. Parmi les autres intérêts de la méthode citons en particulier la flexibilité, il est en effet très facile de généraliser la méthode à des situations physiques complexes, multifluides ([5], [19]), multiphasiques ([20]), incluant éventuellement des effets viscoplastiques, de l'endommagement ([6])... La méthode devient alors très attractive en terme de coût et de performances.

Conservativité. $h$ variable Un élément important s'est rapidement imposé dans toute les applications, c'est la nécessité de faire varier $h$ en fonction de la particule et du temps; cela s'avère indispensable si l'on veut, ne serait ce qu'en dimension un d'espace, calculer la solution d'un tube à choc type Sod. Un tel calcul, mené avec $h$ constant, conduit très rapidement à des instabilités dans la zone de détente. Cela s'explique très simplement; la méthode étant par nature Lagrangienne, les particules s'écartent dans la zone de détente, et en particulier à $h$ constant le nombre de voisin décroit, provoquant une augmentation irrémédiable des erreurs numériques dans les calculs des dérivées. Le remède est simple ([19], voir aussi [5]), il suffit de faire évoluer $h$ localement afin de garder le nombre de voisins le plus proche possible des valeurs optimales. En pratique on modifie les principes d'approximation suivant:

$$
\begin{gathered}
<f(x)>_{g}=\int f(y) W(x-y, h(x)) d y \\
\Pi_{g}^{h}(f)_{i}=\sum_{i \in P} w_{i} f_{i} W\left(x_{i}-x_{j}, h_{i}\right) \\
W_{i j} \equiv W\left(x_{j}-x_{i}, h_{i}\right) \quad \nabla W_{i j} \equiv \operatorname{grad}_{x}\left[W\left(x_{j}-x_{i}, h_{i}\right)\right] \\
\nabla^{h} \Pi_{g}^{h}(f)_{i}=\sum_{j \in P} w_{j} f_{j} \nabla W_{i j}
\end{gathered}
$$

et on utilise les formules habituelles (5) où $\nabla^{h} \Pi^{h}$ est remplacé par $\nabla^{h} \Pi_{g}^{h}(f)$. 
On n'a plus $\nabla W_{i j}=-\nabla W_{j i}$ et donc la conservativité globale n'est plus respectée. C'est une erreur du second ordre qui ne semble pas avoir d'effets néfastes sur les résultats, sauf si l'on s'intéresse précisément à la conservation de quantités macroscopiques comme le moment d'un objet.

Conditions aux limites Cela reste une des difficultés principales des calculs dès que l'on veut effectuer des simulations en enceinte fermée. La technique habituelle utilise des particules fantômes, quelques tentatives ont été menées par Monaghan ([21]) pour les remplacer par des forces de paroi (voir aussi [9]), mais cela reste très empirique.

Contraste A nalyse-Pratique Je terminerai ces commentaires en faisant remarquer que l'on effectue en pratique tous les calculs avec $\frac{h}{\Delta x} \simeq 1.2$ (au moins pour le noyau régularisant donné en exemple et en dimension 2) ce qui nous place très loin des considérations théoriques qui nous amèneraient au minimum à imposer $\frac{\Delta x}{h} \longrightarrow 0$.

Un exemple de calcul complexe A titre d'exemple et pour montrer ce que l'on peut attendre de calculs menés à l'aide de méthodes particulaires de type SPH, je présente ici des résultats de simulations, obtenus avec le code SmartFluid développé par XRS et Simulog. Il s'agit de la désintégration d'un jet liquide (quasi incompressible) par un écoulement gazeux à grande vitesse. Le calcul est tridimensionnel selon le schéma de la figure 2.4.

Trois cas correspondant à différentes vitesses du gaz et du jet sont présentés et comparés à des essais expérimentaux réalisés au CORIA ([28]).

Seules sont représentées dans les images issues du calcul les particules fluides. Les photographies montrent la répartition expérimentale des gouttes de fluide au même instant. On contaste un accord qualitatif assez convainquant entre les données expérimentales et le calcul. Ces résultats sont assez caractéristiques de ce que l'on peut attendre de ces méthodes pour le calcul d'écoulements complexes multifluides. Je renvoi à [15] pour de plus amples détails.

\section{Formulation Faible}

On introduit maintenant une nouvelle formulation de ces méthodes. Elle est basée sur la formulation faible des équations et permet de résoudre positivement les problèmes évoqués précédemment.

\subsection{Principes de base}

On cherche donc à approcher une solution du système (4), mais sous sa forme faible définie par:

$$
\begin{aligned}
& \forall \varphi \in C_{0}^{2}\left(\mathbb{R}^{d} \times \mathbb{R}^{+, *}\right) \\
& \int_{\mathbb{R}^{d} \times \mathbb{R}^{+}}\left(\Phi . L_{v}^{*}(\varphi)+F(x, t, \Phi) \cdot \nabla(\varphi)+S \cdot \varphi\right) d x d t=0
\end{aligned}
$$

$-L_{v}^{*}$ adjoint de $L_{v}$ :

$$
L_{v}^{*}(\varphi)=\frac{\partial \varphi}{\partial t}+\sum_{l=1, d} v^{l} \frac{\partial \varphi}{\partial x^{l}}
$$

On ne s'intéresse pas ici aux problèmes d'existence et d'unicité des solutions liés par exemple aux conditions d'entropie, il est tout a fait possible d'en tenir compte (cf par exemple [2]) mais cela n'a rien d'essentiel pour la compréhension du nouveau formalisme développé ici. 

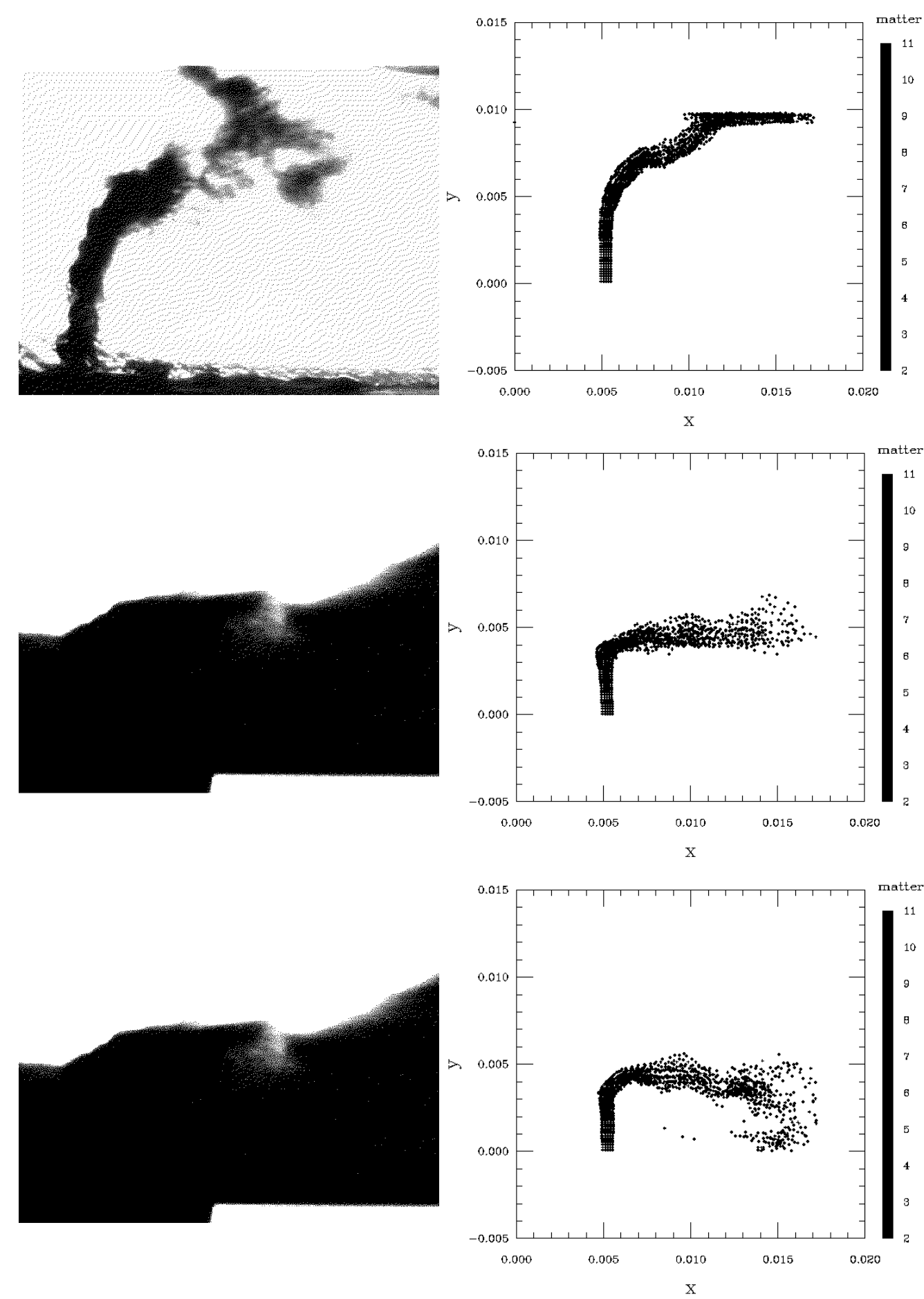

Fig. 1 - Comparaison Expérience-Calcul 


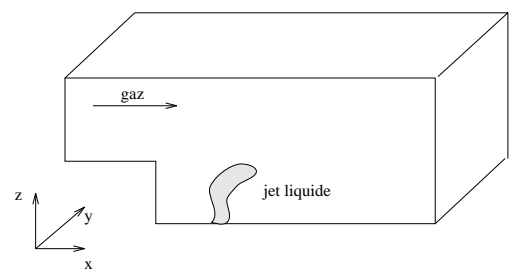

FIG. 2 - Dispositif expérimental

Le passage au discret se fait en trois étapes:

- intégrales sur $\mathbb{R}^{d} \rightarrow$ produit scalaire discret sur les particules:

$$
\int_{\mathbb{R}^{d}} f . g d x \rightarrow(f, g)_{h}:=\sum_{j \in P} w_{j} f_{j} \cdot g_{j}
$$

On demande que pour $f$ et $g$ intégrables il y ait convergence de $(f, g)_{h}$ vers le produit scalaire continu associé, cela est assuré avec un minimum de conditions sur la formule de quadrature associée.

- dérivation continue $\rightarrow D_{h, S}$

$$
\vec{\nabla} f \rightarrow D_{h, S} f
$$

On demande qu'il y ait convergence de $D_{h, S} f$ vers $\vec{\nabla} f$ pour $f$ suffisamment régulière. On introduit l'opérateur adjoint $-D_{h, S}^{*}$

$$
\left(D_{h, S}^{*} f, g\right)_{h}=-\left(f, D_{h, S} g\right)_{h}
$$

- le schéma particulaire est défini en appliquant ces règles de substitution à la formulation faible (9), ce qui donne $\forall \varphi \in C_{0}^{2}\left(\mathbb{R}^{d} \times \mathbb{R}^{+, *}\right)$ :

$$
\int_{\mathbb{R}^{+}}\left[\left(\Phi, L_{v}^{*}(\varphi)\right)_{h}+\left(F(\Phi), D_{h, S} \varphi\right)_{h}+(S, \varphi)_{h}\right] d t=0
$$

On a alors

$$
\begin{aligned}
\int_{\mathbb{R}^{+}}\left(\Phi, L_{v}^{*}(\varphi)\right)_{h} d t & =\int_{\mathbb{R}^{+}}\left(\Phi, \frac{d}{d t}(\varphi)\right)_{h} d t \\
& =-\int_{\mathbb{R}^{+}}\left[\sum_{i \in P} \frac{d}{d t}\left(w_{i} \Phi_{i}\right) \varphi_{i}\right] d t \\
& =-\int_{\mathbb{R}^{+}}\left(\frac{1}{w} \frac{d}{d t}(w \Phi), \varphi\right)_{h} d t
\end{aligned}
$$

et

$$
\begin{aligned}
& -\int_{\mathbb{R}^{+}}\left[\left(\frac{1}{w} \frac{d}{d t}(w \Phi), \varphi\right)_{h}+\left(D_{h, S}^{*} F(\Phi), \varphi\right)_{h}-(S, \varphi)_{h}\right] d t \\
= & -\int_{\mathbb{R}^{+}}\left(\frac{1}{w} \frac{d}{d t}(w \Phi)+D_{h, S}^{*} F(\Phi)-S, \varphi\right)_{h} d t=0
\end{aligned}
$$

qui conduit au schéma

$$
\frac{d}{d t}\left(w_{i} \Phi_{i}\right)+w_{i} D_{h, S}^{*}(F)_{i}=w_{i} S_{i}
$$


oul

$$
\frac{d}{d t}\left(\Phi_{i}\right)+\Phi_{i} \operatorname{div}\left(v_{i}\right)+D_{h, S}^{*}(F)_{i}=S_{i}
$$

Par construction les schémas (10) vérifient ainsi un résultat analogue au Théorème de Lax-Wendroff ([16]) pour les différences finies:

Theorem 1 Si $\bar{\Phi}^{0, h}(x, t)=\sum_{j \in P} w_{j} \Phi_{j}(t) \chi_{B_{i}(t)}(x) \rightarrow \Phi$ p.p. et si $D_{h, S} f \rightarrow \nabla f$ pour $f$ régulière quand les paramètres de discrétisation $h$ et $\Delta x \rightarrow 0$, alors $\Phi$ est solution faible de l'edp modèle (4).

Il reste à définir l'opérateur de dérivation discret $D_{h, S}$. En reprenant le calcul précédent il est facile de voir que le schéma (10) est conservatif (i.e. $\left.\frac{d}{d t}(\Phi, 1)_{h}=(S, 1)_{h}\right)$ dès lors que $1(x)$ est dans le noyau de $D_{h, S}$ (i.e. $D_{h, S} f=\nabla f$ pour $f \in P 0$ ).

On retrouve la formulation standard en prenant:

$$
D_{h, S} f=\nabla \Pi^{h}(f)-f \nabla \Pi^{h}(1)
$$

On a alors

$$
D_{h, S} f_{i}=\sum_{j \in P} w_{j}\left(f_{j}-f_{i}\right) \nabla W_{i j}
$$

avec $P 0 \subset \operatorname{ker}\left(D_{h, S}\right)$ et

$$
D_{h, S}^{*} f_{i}=\sum_{j \in P} w_{j}\left(f_{j}+f_{i}\right) \nabla W_{i j}=\nabla \Pi^{h}(f)_{i}+f_{i} \nabla \Pi^{h}(1)_{i}
$$

Tous les aspects liés au traitement des conditions aux limites rentrent également dans ce formalisme. Je renvoi à [31] pour une analyse détaillée des principes et à [3], [4] pour des résultats de convergence dans le cas scalaire non linéaire. Il est simplement important de noter ici que les différentes méthodes existantes (particules fantômes et forces de paroi) sont à interpréter à partir de termes de bord dans la formulation faible qui produisent ensuite des termes sources volumiques dans l'équation (10) par régularisation des mesures surfaciques correspondantes.

On détaillera plus particulièrement dans ces notes les aspects liés à une utilisation conservative et consistante de la longueur de lissage variable et aux extensions par la technique de renormalisation.

\subsection{Longueur de lissage variable}

Tous les outils sont en place, on dispose d'un formalisme $h$ variable évoqué au paragraphe 2.4:

$$
\begin{gathered}
<f(x)>_{g}=\int f(y) W(x-y, h(x)) d y \\
\Pi_{g}^{h}(f)_{i}=\sum_{i \in P} w_{i} f_{i} W\left(x_{i}-x_{j}, h_{i}\right) \\
W_{i j} \equiv W\left(x_{j}-x_{i}, h_{i}\right) \quad \nabla W_{i j} \equiv \operatorname{grad}_{x}\left[W\left(x_{j}-x_{i}, h_{i}\right)\right]
\end{gathered}
$$

Il s'agit de la formulation "gather" dans la littérature SPH (voir [10]). La seule difficulté est de lui associer un opérateur $D_{h, S}$ linéaire et possédant les propriétés de consistance suffisante. Cela se réalise en introduisant

ESAIM: Proc., VoL. 3, 1998, 131-146 


$$
\nabla^{h} \Pi_{g}^{h}(f)_{i}=\sum_{j \in P} w_{j} f_{j} \nabla W_{i j}
$$

qui n'est pas contrairement au cas standard la dérivée exacte de l'approximation particulaire $\Pi_{g}^{h}(f)$. On peut dans ces conditions démontrer des résultats d'approximation tout a fait similaires à (3). Je renvoi à la thèse de J.L. Lacôme ([12]) pour une étude détaillée ( voir aussi [31], [13]). On définit donc l'opérateur $D_{h, S}$ de la manière suivante:

$$
\begin{aligned}
& D_{h, S} f=\nabla^{h} \Pi_{g}^{h}(f)-f \nabla^{h} \Pi_{g}^{h}(1) \\
& D_{h, S} f_{i}=\sum_{j \in P} w_{j}\left(f_{j}-f_{i}\right) \nabla W_{i j}
\end{aligned}
$$

Il vérifie la propriété de noyau nécessaire à la conservativité et l'opérateur adjoint définissant le schéma est donné par:

$$
D_{h, S}^{*} f_{i}=\sum_{j \in P} w_{j}\left(f_{i} \nabla W_{i j}-f_{j} \nabla W_{j i}\right)
$$

On ne retrouve pas les formules standards (sauf si $\nabla W_{i j}=-\nabla W_{j i}$ ) mais on est conservatif par construction.

\subsection{Renormalisation}

La réponse concernant $h$ variable, donnée à la section précédente est satisfaisante, mais ne permet pas de combler le trou entre la pratique et la théorie, lié aux estimations d'erreur qui sous entendent que le rapport $\frac{(\Delta x)}{h} \rightarrow 0$. Le concept de renormalisation de l'approximation particulaire est apparu récemment dans la littérature SPH ([24]). On va montrer que, employé à bon escient avec le formalisme précédent, il permet d'envisager des méthodes plus robustes et de relaxer la condition sur le ratio $\frac{(\Delta x)}{h}$.

L'idée de renormalisation (exprimée dans ce cadre formel) est assez simple, il s'agit de modifier les formules définissant l'opérateur $D_{h, S} f(x)$ à l'aide d'une matrice poids (ou de renormalisation ) $B(x)$ de la façon suivante:

$$
D_{h, S} f(x)=B(x) \cdot \nabla^{h} \Pi_{g}^{h}(f)(x)-f(x) B(x) \nabla^{h} \Pi_{g}^{h}(1)(x)
$$

On cherche à augmenter la précision, il est alors naturel de demander non plus $P 0 \subset$ $\operatorname{ker}\left(D_{h, S}\right)$ mais $P 1 \subset \operatorname{ker}\left(D_{h, S}\right)$. On démontre très facilement la

Proposition 2 On a $P 1 \subset \operatorname{ker}\left(D_{h, S}\right)$ ou de manière équivalente $D_{h, S} f=\nabla f$ pour tout polynôme $f$ de degré 1 au plus, si et seulement si $B(x)=E(x)^{-1}$ avec

$$
E(x)^{\alpha \beta}=\sum_{j \in P} w_{j}\left(x_{j}^{\beta}-x^{\beta}\right) \partial^{\alpha} W\left(x, x_{j}\right)
$$

On a alors

$$
E(x)^{\alpha \beta}=\partial^{\alpha, h} \Pi_{g}^{h}\left(x^{\beta}\right)-x^{\beta} \partial^{\alpha, h} \Pi_{g}^{h}(1)
$$

L'extention évoquée au paragraphe précédent des estimations (3) assure alors que $E(x)^{\alpha \beta} \simeq$ $\delta^{\alpha \beta}$ et donc que $B(x)$ est définie, sous l' hypothèse $\frac{(\Delta x)}{h} \rightarrow 0$. On a la propriété suivante:

Proposition 3 Sous l'hypothèse B uniformément bornée on a 


$$
\left\|D_{h, S} \varphi(x)-D \varphi(x)\right\| \leq C h_{0}\|B(x)\|\left\|D^{2} \varphi\right\|_{\infty}
$$

où $h_{0}$ est l'échelle caractéristique le la longueur de lissage (i.e. $\eta_{-} h_{0} \leq h \leq \eta_{+} h_{0}$ avec $\eta_{-}$et $\eta_{+}$deux constantes $>0$ )

La consistance de la méthode est donc assurée dès lors que la longueur de lissage tend vers zéro. On peut en fait montrer si $\frac{\Delta x}{h}$ est borné et si la distribution initiale de particule est régulière (condition sur la formule de quadrature) que la matrice $B(x)$ est uniformément bornée et que:

$$
\left|\left(\varphi, D_{h, S}(\varphi)\right)_{h}\right|=\left|\left(\varphi, D_{h, S}^{*}(\varphi)\right)_{h}\right| \leq C\|\varphi\|_{h}^{2} .
$$

ce qui assure le résultat d'approximation et qui permet également de montrer la stabilité de la méthode dans le cas linéaire (système) ainsi que sa convergence. les opérateurs de dérivation discrète sont alors définis selon:

$$
\begin{gathered}
D_{h, S} f_{i}=\sum_{j \in P} w_{j}\left(f_{j}-f_{i}\right) B_{i} . \nabla W_{i j} \\
D_{h, S}^{*} f_{i}=\sum_{j \in P} w_{j}\left(f_{i} B_{i} . \nabla W_{i j}-f_{j} B_{j} . \nabla W_{j i}\right)
\end{gathered}
$$

et le schéma par:

$$
\frac{d}{d t}\left(w_{i} \Phi_{i}\right)+w_{i} \sum_{j \in P} w_{j}\left(f_{i} B_{i} . \nabla W_{i j}-f_{j} B_{j} . \nabla W_{j i}\right)=w_{i} S_{i}
$$

On renvoit à [29] pour une présentation détaillée de ces résultats.

Nous présentons néanmoins ( $\mathrm{cf}$. figure 3.3) quelques résultats numériques évocateurs obtenus par N. Lanson sur un problème simple comme le tube à choc de Sod. On compare içi les méthodes standard ( $h$ variable conservatif ) à la méthode renormalisée (on renvoit à [1],[14] pour les détails des algorithmes effectivement utilisés) pour différentes valeurs de $\frac{\Delta x}{h}$. La méthode renormalisée est effectivement insensible à la valeur du ratio $\frac{\Delta x}{h}$. utilisé alors que la méthode usuelle ne donne les bonnes valeurs des niveaux dans la solution du problème de Riemann que pour des valeurs de $\frac{\Delta x}{h}$ proches de 0.5 .

L'interprétation du résultat est la suivante: un calcul très simple montre que pour la valeur $\frac{\Delta x}{h}=0.5$ et sur un maillage uniforme l'approximation $\nabla \Pi^{h}(x)$ de 1 est exacte, le bon comportement de la méthode standart (où modifiée en $h$ variable) en résulte pour les valeurs du ratio proche de cette valeur optimale. Il est à noter que cette valeur optimale depend bien entendu du choix du noyau régularisant ainsi que de la dimension de l'espace.

Outre sa robustesse et son insensibilité à la variation locale de $h$, la méthode renormalisée permet de diminuer le nombre de voisins effectifs d'une particule donnée. Les test effectués en dimension deux d'espace montrent que 8 voisins sont suffisants, ce qui diminue de plus de moitié le coût de la méthode.

\subsection{Schémas Hybrides Volumes Finis - Particulaires}

Je reviens enfin à un cadre plus classique, en montrant comment on peut inclure des schémas type Godunov issus de la méthodologie Volumes finis dans le cadre des méthodes présentées ici. On se place dans le cas d'un noyau W à symétrie sphérique:

$$
\begin{aligned}
\nabla_{x} W\left(x_{i}-x_{j}\right) & =-D \theta_{i j} n_{i j} \quad n_{i j}=\frac{x_{j}-x_{i}}{\left\|x_{j}-x_{i}\right\|}, \\
D \theta_{i j} & =D \theta\left(\left\|x_{i}-x_{j}\right\|\right) \leq 0
\end{aligned}
$$

ESAIM: Proc., VoL. 3, 1998, 131-146 

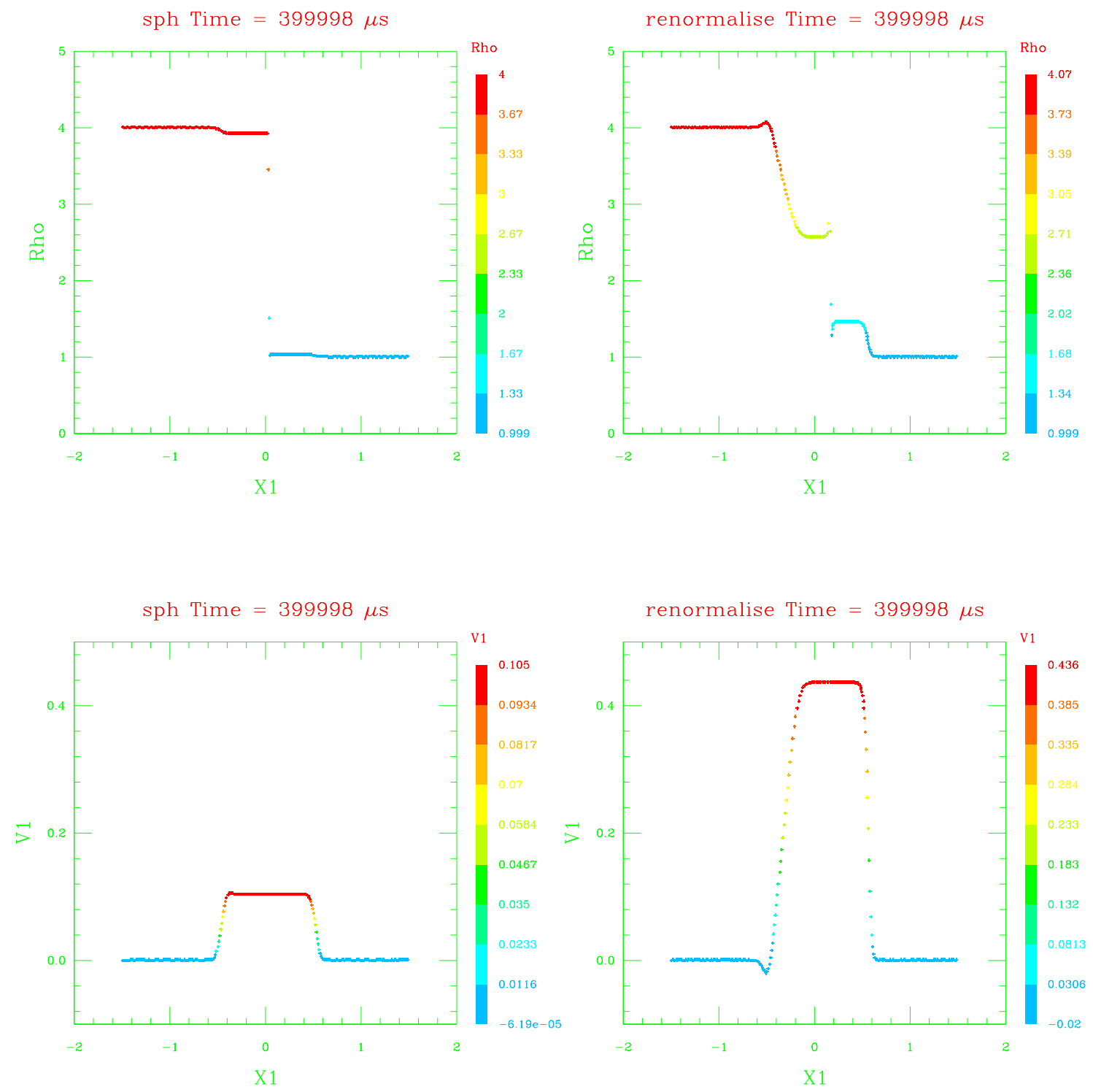

FIG. 3 - Comparaison SPH-Renormalisé $\frac{\Delta x}{h}=1.666$ 
Prenant pour simplifier $S=0$, on obtient le schéma suivant

$$
\frac{d}{d t}\left(w_{i} \Phi_{i}\right)-w_{i} \sum_{j \in P} w_{j}\left(F_{i}+F_{j}\right) . n_{i j} D \theta_{i j}=0
$$

qui fait apparraitre une loi de conservation selon la direction $n_{i j}$ :

$$
\frac{\partial}{\partial t}(\Phi)+\frac{\partial}{\partial x}\left(F\left(x_{i j}, t, \Phi\right) \cdot n_{i j}\right)=0
$$

Il est alors tout a fait naturel d'introduire en lieu et place de $\left(F\left(\Phi_{i}\right)+F\left(\Phi_{j}\right)\right) . n_{i j}$, le flux d'un schéma Volumes Finis $2 g\left(n_{i j}, \Phi_{i}, \Phi_{j}\right)$ satisfaisant à

$$
\begin{aligned}
& \text { (i) } \quad g(n, u, u)=F(u) . n \\
& \text { (ii) } g(n, u, v)=-g(-n, v, u)
\end{aligned}
$$

et donc associé à la loi de conservation (12). La viscosité numérique du schéma est classiquement définie par

$$
Q(n, u, v)=\frac{F(u) \cdot n-2 g(n, u, v)+F(v) \cdot n}{v-u}
$$

Le nouveau système s'écrit donc

$$
\begin{aligned}
\frac{d}{d t}\left(w_{i} \Phi_{i}\right)-w_{i} \sum_{j \in P} w_{l} 2 g\left(n_{i j}, \Phi_{i}, \Phi_{j}\right) D \theta_{i j} & =0 \\
\Phi_{i}(0) & =\Phi^{0}\left(\xi_{i}\right)
\end{aligned}
$$

ou encore

$$
\begin{aligned}
& \frac{d}{d t}\left(w_{i} \Phi_{i}\right)-w_{i} \sum_{j \in P} w_{j}\left(F\left(x_{i j}, t, \Phi_{i}\right)+F\left(x_{i j}, t, \Phi_{j}\right)\right) . n_{i j} \\
& \Phi_{i}(0)=\Phi^{0}\left(\xi_{i}\right)^{+Q\left(n_{i j}, \Phi_{i}, \Phi_{j}\right)\left(\Phi_{i}-\Phi_{j}\right) D \theta_{i j}=0}
\end{aligned}
$$

expression qui est tout a fait analogue à l'expression (7) classique en $\mathrm{SPH}, Q\left(n_{i j}, \Phi_{i}, \Phi_{j}\right)$ $\left(\Phi_{i}-\Phi_{j}\right)$ remplaçant ici $\Pi_{i j}$. On peut attendre de cette approche plus de robustesse comme c'est le cas en Volumes Finis. L'analyse mathématique de la convergence est possible dans le cas scalaire non linéaire à l'aide des techniques maintenant classiques utilisant les solutions mesures des lois de conservation (cf [2] ).

Ce type d'approche permet également un couplage efficace entre méthodes VF et particulaires, il permet également en disposant du champ de transport $v^{0}(x, t)$ de construire des méthodes particulaires de type ALE (Arbitrary Lagrange Euler). On renvoit à ([30], [22]) pour une description plus détaillée dans le cadre des équations d'Euler et des exemples d'applications.

\section{Conclusion}

J'ai essayé dans cet article de poser quelques principes relatifs au développement de méthodes particulaires régularisées et plus particulièrement de type SPH. Ce sont des méthodes numériques peu connues dans la communauté des Mathématiques Appliquées et dans la communauté habituelle de la Mécanique des Fluides. Les applications qu'elles permettent de traiter, leur souplesse, les rendent très attractives. C 'est ègalement un champ de recherche intéressant pour le mathématicien applliqué, bon nombre de problèmes théoriques et pratiques sont encore à débroussailler et peuvent faire l'objet de collaborations fructueuses avec des partenaires physiciens ou industriels.

ESAIM: Proc., VoL. 3, 1998, 131-146 


\section{Références}

[1] B. Ben Moussa, N. Lanson \& J.P. Vila, Gridless methods for conservation laws, application to Euler equations, (en préparation).

[2] B. Ben Moussa \& J.P. Vila, Convergence of SPH method for scalar nonlinear conservation laws, Preprint MIP, 1996.

[3] B. Ben Moussa, PHD thèse INSA Toulouse MIP, 1997.

[4] B. Ben Moussa \& J.P. Vila, Convergence of SPH method for scalar nonlinear conservation laws on bounded domains, Rapport interne MIP (à paraitre).

[5] W. Benz, Smooth Particle Hydrodynamics : a Review. Harvard-Smithsonian Center for Astrophysics, Preprint 2884, 1989.

[6] W. Benz \& A. Asphaug, Impact Simulations with Fracture: I. Methods and Tests, Icarus, 1993.

[7] R.A. Gingold \& J.J. Monahghan, MNRAS, (1977), 181-375.

[8] R.A. Gingold \& J.J. Monahghan, Shock simulation by the particle method, S.P.H., J.C.P. 52, (1983), 374-389.

[9] Herant, Dirty Tricks for SPH, Mem. S. A. It., Vol. XX, 1993.

[10] L. HERnquist \& N. Katz, TREESPH : a unification of SPH with the hierarchical tree method, The Astr. J. S.S., 70, (1989). 419-446.

[11] G.R. Johnson, Artificial viscosity effects for SPH computations, Int. J. Impact Engng Vol 18, 5, (1996), 477-488.

[12] J.L. Lacôme, Thèse INSA Toulouse MIP CEG, 1997.

[13] J.L. LACôME \& J.P. ViLA, en préparation.

[14] N. Lanson, Rapport de DEA juin 97, MIP INSAT.

[15] E. Larrey, G. Vich, A. Merlo, M. Ledoux, A. Rompteaux \& J.P. Vila, Liquid Jet disintegration in a gas stream, ICLAS, Seoul, 1997

[16] P.D. Lax \& B. Wendroff, Systems of Conservation Laws, CPAM, 13, (1960), 217237.

[17] L. LuCY, Astrono. J., 82, (1977), 1013.

[18] S. Mas-Gallic \& P.A. Raviart, A Particle Method for First-order Symmetric Systems, Numer. Math. 51, (1987), 323-352.

[19] J.J. Monaghan, Smooth Particle Hydrodynamics, Annu. Rev. Astron. Astrop. 30, (1992), 543-574

[20] J.J. Monaghan \& Kocharyan, SPH simulation of multi-phase flow, Comp. Physics Comm. 87, (1995), 225-235.

[21] J.J. Monahghan, Lecture Notes, Kaiserslautern University, 1995.

[22] L. Paris, Rapport de Fin d'étude, INSA GMM, 1996.

[23] A.G. Petschek \& L.D. Libersky, Cylindrical Smooth Particle Hydrodynamics, $J$. Comp. Phys. 109, (1993), 76-83.

[24] R.W. Randles \& L.D. Libertsky, Smoothed Particle Hydrodynamics, Some recent improvements and Applications, Comp. Meth. Appli. Mech. Eng;, 139, (1996), 375-408.

[25] P.A. Raviart, An analysis of particle methods, in Num. method in fluid dynamics, F. Brezzi ed.. Lecture Notes in Math., vol 1127, Berlin, Springer, 1985.

[26] P.A. Raviant, Particle approximation of first order systems, Journal of Comp. Mathematics, Vol 4, 1, 1986. 
[27] R.D. Richtmeyer \& K.W. Morton, Difference methods for initial value problems, Interscience Publishers, New York, 1967.

[28] G. Vich \& M. Ledoux, Disintegration of a liquid jet by a subsonic crossflow, ICLAS, Seoul, 1997.

[29] J.P. VILA, Gridless Methods for Conservation Laws, Rapport MIP, juin 1997.

[30] J.P. Vila, Weighted Particle-Finite Volume Hybrid schemes, in Finite Volumes for Complex Applications, ed. F. Benkhaldoun, R. Vilsmeier, HERMES, Paris, 1996.

[31] J.P. VILA, Particle Weighted methods and Smooth Particle Hydridynamics, (soumis à M3AS). 